\title{
Creation Nets: Harnessing The Potential Of Open Innovation
}

John Hagel III, Co-Chairman, Deloitte LLP Center for Edge Innovation, USA John Seely Brown, Independent Co-Chairman, Deloitte LLP Center for Edge Innovation, USA

\section{INTRODUCTION}

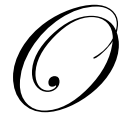
pen innovation has become the latest management buzzword. We would have to look long and hard to find an executive who does not agree with the basic premise of open innovation - that success in coming up with creative new value to offer to the marketplace depends upon connecting effectively with other companies. Executives realize they simply can't do it alone. Virtually all of them now buy into the wisdom of Bill Joy's observation that "there are always more smart people outside your company than within." i

But here's the paradox: despite such broad acceptance of the basic premise of open innovation, why are there so few examples of companies that systematically and successfully practice this approach? Any discussion of open innovation will offer inspiring stories from Procter \& Gamble, Cisco, and Eli Lilly, but these are the same stories told over and over again. Why do so few companies actually succeed in the broad based implementation of this important management principle?

At least two major obstacles prevent companies from achieving greater success with open innovation. First, there is a lot of confusion over what open innovation actually involves. Mention open innovation and many executives immediately jump to extreme examples like open source software. With this image in mind, the doubts and anxieties rapidly bubble to the surface. Will we lose control? What happens to intellectual property? Does anyone ever make money from these kinds of initiatives? At the other extreme, executives think much too narrowly about open innovation. They might point to a joint venture with a key business partner that produced an important new product or to a licensing deal providing access to a new technology. Using these examples, they proudly announce they have mastered open innovation, even though these were one-off efforts with limited impact on the overall performance of the business.

Second, there is further confusion regarding the management techniques required to deliver continuous value from open innovation initiatives, especially when they involve hundreds, if not thousands, of business partners. If you read many of the champions of open innovation, they describe these efforts in ways that shed little light on the management methods required to harness the potential of these initiatives. In fact, by using terms like "emergent" or "self-organizing," these champions tend to give the impression that these efforts cannot really be managed, that they acquire a life of their own beyond the reach or influence of any individual company.

In fact, these two obstacles are related. Fear about open innovation often stems from lack of clarity regarding the management techniques that can be used to focus these efforts and keep transaction costs down. On the other hand, without clarity regarding management techniques that can effectively mobilize large numbers of business partners, executives are likely to fall back to very narrow ways of thinking about open innovation, generating a sense of complacency and a conviction that traditional management techniques are more than sufficient to generate value.

These obstacles are understandable. Even though the roots of open innovation go at least as far back as the Italian Renaissance, today's variants on the model are anything but mainstream. They are emerging and thriving on the peripheries and edges of most of our experience, in such unexpected areas as China, on the big wave surfing beaches of Hawaii, among the thousands of telescopes spread around the world manned by amateur astronomers and 
tied together by the Internet, and in the coding efforts of thousands of programmers coming together online in various open source software initiatives.

If we look at the patterns emerging on these peripheries, we begin to see a new way of organizing and managing open innovation. Hundreds and, in many cases, thousands of participants are coming together in distributed creation nets assembled by network organizers. These network organizers use a distinctive set of management techniques to ensure focus and value creation. By understanding these new ways of organizing open innovation, executives can finally close the gap between the potential of open innovation and the actual value generated from such initiatives.

\section{CHARACTERISTICS OF CREATION NETS}

The term "open innovation" has been used with many different meanings. Henry Chesbrough, who literally wrote the book on Open Innovation ${ }_{-}^{\mathrm{ii}}$, defines it broadly as "a paradigm that assumes that firms can and should use external ideas, and internal and external paths to market, as firms look to advance their technology." Under this definition, many different kinds of initiatives fall under the rubric of open innovation: scanning the external environment for ideas; reaching out to a specialist on a contract basis to solve a particularly vexing research problem; forming a joint venture; licensing technology from a university; or participating in broad networks to coordinate innovation activity.

Creation nets represent a particularly powerful form of open innovation designed to harness the potential of distributed innovation activity pursued by hundreds or thousands of participants. Creation nets implement a set of institutional mechanisms designed to mobilize independent entities in the pursuit of distributed, collaborative, and cumulative innovation. These institutional mechanisms are critical to understanding how creation nets coordinate innovation efforts and how these creation nets will re-shape the role and structure of the firm.

These networks are assembled by a network organizer who serves as gatekeeper, deciding who will be able to participate in the network. The network organizer also defines fundamental governance processes to coordinate the activities of the network, for example, determining how disputes will be resolved and how performance will be measured. These participation protocols are generally simple and informal, especially in the early stages of network formation. Participation in the network is rarely established through formal contractual documents, although specific initiatives within the network may be governed by such contracts.

The network organizer could be either an individual or small core team, as in the case of many open source software initiatives, or it could be a corporation or other form of institution. While the network organizer serves in some respects as the hub of the network, the relationships within the network are usually far more complex than a simple hub and spoke model would suggest.

Creation nets typically organize their activities into modular processes (although, as we will see, the degree of process focus differs depending on the type of creation net), making it much easier to incorporate a large number of participants. This modular approach also leaves a lot of freedom to participants to innovate within their own module of activity while ensuring that interfaces across modules are well-defined so that activity across modules can be coordinated. For this reason, creation nets focus on defining performance requirements rather than specifying individual activities within modules. The modular approach to processes makes it feasible for many participants to innovate in parallel and simultaneously pursue a variety of different approaches to delivering the required performance. More broadly, these modular management approaches represent examples of innovative "pull" approaches to resource mobilization in contrast to more conventional "push" approaches ${ }^{\text {iii. }}$.

While creation nets are loose on one dimension, they are remarkably tight on another dimension. They are very adept at defining clear "action points" where participants must come together and deliver outputs. Where inconsistencies or incompatibilities exist, participants must make clear choices in order to produce an integrated product or offer for use by others. 
Creation nets also rely on long-term incentive structures to motivate and align participants. To be sure, many creation nets are explicitly commercial and reward participants with cash or contracts for delivering successful innovations. But even in these commercial creation nets, participants find that the real rewards are longer-term. By joining these networks, participants find that they are able to get better faster by working with others in the networks rather than working on their own. For this reason, they are motivated to do the right thing in the near-term rather than pursuing opportunistic short-term profit maximization. Successful creation nets must therefore focus on building long-term relationships with participants, creating opportunities for repeated interactions that demonstrate the value of cooperation.

While creation nets share these characteristics in common, there are also significant differences across creation nets. One of the most significant differences involves the degree of diversity among participants.

Some of the most well-known examples of creation nets - for example, open source software initiatives or extreme sports networks - bring together participants who share a relatively common set of practices. These creation nets are able to rely on looser forms of coordination and we categorize this sub-set as "practice networks." Because the participants in these networks share a common sensibility and set of practices, the organizers of these networks we call them practice orchestrators - can play a less active role, both in terms of recruitment and management of specific creation initiatives. These practice orchestrators tend to focus much more on the integration stage of the creation process when the contributions of the participants must be brought together and incorporated into a consistent or compatible release or offering.

Other types of creation nets - for example, the design networks assembled by original design manufacturers (ODMs) in Taiwan or the production network created by Li \& Fung in China - mobilize participants with very different practices and experiences. As a result, these require more active forms of coordination (although still far looser than most executives of large enterprises would be comfortable with). We categorize this sub-set as "process networks" - an innovative type of organization to tap into distributed expertise that we have written about extensively in the past ${ }^{\text {iv }}$. As we shall see below, understanding which sub-set of creation networks is most appropriate will help to tailor the right set of management techniques.

\begin{tabular}{|l|l|l|}
\hline & \multicolumn{1}{|c|}{ Global process networks } & \multicolumn{1}{c|}{ Global practice networks } \\
\hline Types of participants & Highly diverse specializations & Similar sensibilities and practices \\
\hline Examples & $\begin{array}{l}\text { ODM product design networks } \\
\text { Li \& Fung supply chain and design } \\
\text { networks }\end{array}$ & $\begin{array}{l}\text { Open source software initiatives } \\
\text { Extreme sports networks }\end{array}$ \\
\hline Type of network organizer & Process orchestrator & Practice orchestrator \\
\hline Primary roles of organizer & $\begin{array}{l}\text { Vetting of participants } \\
\text { Assignment of roles } \\
\text { Monitoring of performance } \\
\text { Providing performance feedback }\end{array}$ & $\begin{array}{l}\text { Definition of protocols for } \\
\text { participation } \\
\text { Definition of protocols for integration } \\
\text { activity }\end{array}$ \\
\hline
\end{tabular}

\section{THE DISTINCTIVE POWER OF CREATION NETS}

If we look at historical periods and geographic regions characterized by significant economic growth, we certainly find bright individuals and innovative organizations, but we also find something else. These individuals and organizations come together and collaborate in evolving networks of creation, or creation nets. They play off each other, appropriating each other's work, learning from it, building on top of it and then watching and learning from what others do with their own creations. They often work in parallel and then fight and learn from each other when the time comes to try to integrate their work into a broader offering. The relationships that they establish are usually something more than arms length commercial relationships but also something less than formal joint ventures.

Creation nets have emerged and flourished throughout history, with their center of gravity flowing broadly westward from Renaissance Italy to eighteenth century England, nineteenth century New England, twentieth century 
Silicon Valley and now to twenty-first century Asia. In these periods, exploration and innovation become more valuable than achieving greater efficiency in existing operations. Established institutions are often challenged in these periods and find it difficult to adapt to rapid and widespread change. Creation nets emerge as catalysts in these periods to re-shape and re-focus institutions.

Rapid change has a profound impact on the sources of economic value. In times of relative stability, what we uniquely know - our "stock" of distinctive knowledge - is extremely valuable and needs to be carefully protected. Since the environment is changing relatively slowly, this knowledge can continue to create value indefinitely. If others acquire this knowledge, they can use it to compete directly with us. By using this knowledge, they threaten to erode our distinction in the market place - we are in a zero sum world - so it pays to be extremely protective of our stocks of knowledge. We seek to protect this knowledge as long as possible, lobbying with governments to strengthen intellectual property laws and extend the period of exclusivity wherever feasible.

As change accelerates, something interesting happens - and it can be very unsettling to leaders of large, established institutions. All of a sudden, what we know - those "stocks" of knowledge - becomes less valuable. The life-time value of knowledge rapidly shrinks as the rate of obsolescence in knowledge increases. Now, the game becomes using our knowledge as a way to connect more rapidly and effectively with others to create new knowledge. Stocks of knowledge become progressively less valuable while flows of knowledge - the relationships that can help to generate new knowledge - become more and more valuable. Rather than jealously protecting existing stocks of knowledge, institutions need to offer their own knowledge as a way to encourage others to share their knowledge and help to accelerate new knowledge building.

Now, of course, knowledge does not "flow" - it tends to be, in fact, very "sticky," especially outside very narrowly defined communities of practice. Unlike information, which can be more readily codified and disseminated, knowledge tends to reside in individuals and it is very context specific. For this reason, knowledge sharing typically requires trust-based relationships and a sharing of practice ${ }^{\mathrm{v}}$. Arms length market transactions work great when existing products or codified information are at stake; they are much less useful in accessing, valuing and leveraging knowledge. Knowledge-sharing is amplified when these relationships are grounded in some form of collaborative creation effort. Knowledge-sharing and shared understanding become even more effective when participants come together on a regular basis to undertake new collaborative creation efforts. Thus, creation nets, with their focus on building long-term relationships around sustained collaborative creation efforts and concrete action points, become very powerful vehicles for the catalyzing and participating in flows of knowledge.

Creation nets become important for another reason. During times of rapid change, relevant talent tends to be more dispersed. In more stable times, successful institutions tend to recruit and retain relevant talent. This talent is often highly concentrated in a relatively limited number of institutions.

As change accelerates, peripheries or edges become more important, especially in terms of generating new talent and new knowledge. These edges can take many forms. They may be the edges of existing enterprises, industries, regional economies or even demographic groups. These edges often experience change first and people at these edges are often the first to develop new knowledge in terms of how to deal successfully with these changes. To the extent that these people are affiliated with institutions, they tend to be smaller and more fragmented institutions.

So, knowledge flows become more important in times of rapid economic change and these knowledge flows tend to be much more dispersed than in more stable times. Conventional one-to-one business partnerships or joint ventures are less useful as change accelerates - the trick is to participate in as many relevant flows of knowledge as possible. Once again, creation nets, by creating highly scalable networks of relationships across a broad range of specialized participants, become particularly valuable in times of rapid change.

These creation nets are generally organized first by institutions and individuals on the periphery. With a few notable exceptions, larger institutions tend to be more complacent, especially at the outset, about the implications of accelerating change. They put more value on stocks of knowledge and established positions. In contrast, individuals and institutions playing on the edge see a greater need to form new relationships. They have fewer resources and they see first-hand, often earlier than others, the need to build new knowledge and create new offerings. While larger institutions tend to be in denial about the need for change or view it as a threat, institutions 
and individuals on the periphery see only opportunity. Necessity leads them to adopt a bootstrapping mindset and to seek out or assemble their own creation nets to leverage and accelerate their own efforts.

In practice, creation nets are powerful mechanisms for bootstrapping capability. This is why creation nets have historically played a key role in driving economic development - whether in Renaissance Italy or in England and the United States in the early stages of the Industrial Revolution. It is no accident that they are increasing in importance as the global economy accommodates the entrance of billions of new participants as major national economies - China, India, and the former Communist bloc countries - re-integrate into the global economy and seek to accelerate their own economic development.

But these creation nets are no longer just relevant to entrepreneurial institutions seeking to bootstrap their way off the periphery and into the center of activity. Accelerating change is working its way into the most developed economies and industries, forcing even the largest institutions to rethink their approaches to managing creation and innovation. In a certain sense, everybody is a "developing nation" in a world of rapid change, and everyone needs to learn to bootstrap more aggressively to stay ahead of the game.

In fact, some of the most sophisticated companies are beginning to realize how sources of strategic advantage shift during times of rapid change. Strategic advantage becomes less focused on ownership of distinctive stocks of knowledge. Increasingly, as we have argued in greater depth in The Only Sustainable Edge $e^{\mathrm{vi}}$, advantage resides in the institutional capacity to get better faster - it is not just about the pace of capability building but the relative pace and the ability to accelerate this pace over time. This in turn depends upon privileged access to the most promising flows of knowledge. Existing stocks of knowledge remain important in terms of earning the right to participate in these flows but there is also a need to learn how to evaluate available flows, more effectively participate in them, and more rapidly integrate new knowledge acquired from these flows.

Looked at from this perspective, creation nets are not only useful ways to tap into distributed talent, they become central to building strategic advantage for all institutions. These creation nets are not just learning architectures, but new forms of competitive architecture that can accelerate capability building. In more stable times, operational scale within the firm was a powerful strategic weapon because it enabled companies to move down the learning curve more rapidly than smaller competitors. In more rapidly changing times, learning faster than others will depend upon the scale and scope of creation nets and the ability to participate effectively in these networks.

\section{DIFFERENT INSTITUTIONAL ARCHITECTURES}

Current institutional architectures - business, educational, government, and NGOs - do not map well to these evolving creation nets. Institutional leaders must find ways to build or participate in relevant creation nets to generate more value for their stakeholders. Institutions that choose to remain outside these networks will find it increasingly more challenging to keep up.

In the business world, creation activities tend to remain tightly bundled within individual enterprises. In order to effectively mobilize specialized capability across institutional boundaries, these networks operate very differently relative to more traditional creation initiatives within the enterprise.

\begin{tabular}{|l|l|l|}
\hline & Enterprise & Creation nets \\
\hline Goals & Mixed - routinization and innovation & $\begin{array}{l}\text { Focused - creation of new goods and services } \\
\text { tailored to rapidly evolving market needs }\end{array}$ \\
\hline Scope & Few institutional participants & Multiple institutions and individuals \\
\hline Location & Highly concentrated & More dispersed \\
\hline Innovation approach & Sequential innovation & Parallel innovation \\
\hline Coordination devices & Stage gate reviews & Integration events \\
\hline Governance mechanisms & Process manuals & "Constitutions" and norms \\
\hline Outcome definition & Detailed blueprints & High level performance specs \\
\hline Mobilization techniques & Push & Pull \\
\hline Feedback mechanisms & $\begin{array}{l}\text { Performance reviews and } \\
\text { compensation }\end{array}$ & Appropriation and reuse \\
\hline
\end{tabular}


These differences enable creation nets to become much more powerful engines of innovation, especially where systemic and rapid innovation is needed. In conventional economic theory, high interdependency and speed called for innovation to be concentrated within a single firm. Firms, however, have powerful inertial forces, not the least of which is the continuing need to balance the quest for efficiency and routinization with the requirement to rapidly innovate.

Creation nets establish a much more focused vehicle for driving innovation - the creation of new knowledge, products, and services is their sole rationale for existence. As a result, they place much higher priority on accessing specialized capability wherever it resides. These networks also employ a variety of approaches to engage in creation faster, for example by making it possible for participants to move in parallel wherever possible. At the same time, these creation nets also seek to accelerate appropriation and use of new knowledge, products, and services so that rapid iteration can lead to faster learning. By focusing so aggressively on new knowledge, product, or service creation, these networks also generate significant process innovation as network participants strive to improve their performance.

As a result, creation nets accelerate innovation across their participants. Not only are participants able to innovate more rapidly than they could outside these networks, the pace of their innovation accelerates the longer they participate in the network. This accelerating pace of innovation helps to expand the total value created by the network and reduces many of the risks like opportunism and holdup associated with collaboration in more static environments.

\section{DIFFERENTIATING CREATION NETS FROM OTHER FORMS OF OPEN INNOVATION}

The distinctive approaches to organizing creation nets differentiate these efforts from many other forms of open innovation. For instance, while joint ventures and other more formal collaboration efforts are certainly examples of open innovation, they typically involve a much more limited number of participants, often just two companies coming together in a tightly defined relationship. In fact, the more formal and tight definition of respective activities of the participants makes it very difficult to scale these forms of open innovation. The complexity overhead of trying to manage a large number of diverse participants simply becomes prohibitive.

At another level, creation nets focus on establishing long-term relationships across participants rather than relying on short-term transactions to access external innovation capability. Henry Chesbrough in his book Open Innovation cites examples like IBM licensing intellectual property from third parties. And many observers have discussed the efforts by Eli Lilly to create InnoCentive, an innovation marketplace designed to connect researchers within large companies pursuing difficult research challenges with external experts who can suggest creative approaches to addressing these challenges. These are certainly promising examples of open innovation, but they typically involve short-term transactions between two participants. Even though efforts like InnoCentive require development of long-term institutional frameworks and skill sets, they do not tap into the longer-term incentive structures driving creation nets. Participants are driven by the opportunity to maximize short-term cash value of their efforts rather than seeing a potential to get better faster by working with others.

Other analysts of open innovation, most notably Eric von Hippel in his book Democratizing Innovation ${ }^{\text {vii }}$ and C.K Prahalad and Venkat Ramaswamy, in their book The Future of Competition ${ }^{\text {viii }}$, have placed great emphasis on the opportunities associated with innovation communities or co-creation networks mobilizing customer insight and capability. Once again, these represent interesting opportunities for open innovation, but they tend to focus narrowly on one type of participant: customers. In contrast, creation nets mobilize a broader range of participants including, as appropriate, specialized technology providers, talented hobbyists, manufacturing experts, and suppliers - as well as customers. By reaching out to a broader range of expertise and experience, creation nets can often come up with more innovative approaches than does relying solely on customers as a source of innovation.

Mohanbir Sawhney, in an article on "Communities of Creation: Managing Distributed Innovation in Turbulent Markets" "ix took yet another slice of open innovation, focusing on open source types of innovation. A later book by Steven Weber ${ }^{\mathrm{x}}$, provides an even more detailed view of how open source projects come together and deliver complex software to the market. Both Sawhney and Weber have interesting insights on how open source 
efforts work, but these tend to reinforce the biases of many executives that open innovation undermines commercial business models. While open source initiatives represent one form of creation nets (the practice networks we described earlier), they are not the only type of these networks. In fact, many creation nets are highly successful commercial collaborations that generate significant economic value in the near-term while enabling participants to get better faster and become even more successful commercial enterprises over the long term.

More generally, virtually all the analysts of open innovation tend to focus on product innovation rather than process innovation. In fact, creation nets are powerful engines of both forms of innovation. Some creation nets are explicitly focused on product innovation but, even in these, the modular design of the product design activities provides opportunity for significant innovation in design processes. Even more broadly, many creation nets are focused on the manufacturing of products like apparel or consumer electronics devices or mobilizing the right resources to serve individual customer needs. These creation nets become fertile ground for process innovations, both at the level of individual participants and at the level of the overall process spanning across multiple participants.

\section{AMPLIFIED CAPABILITY OF TODAY'S CREATION NETS}

Creation nets have always played an important role in driving major new waves of economic growth but something is different today. These creation nets are becoming even more powerful engines of innovation and capability building. Thanks to new generations of technology and an emerging global digital infrastructure, rapid cycles of distributed innovation and talent development are becoming more feasible. In addition to accommodating more participants from more locations, these technologies are also increasing the speed of creation. The compression of cycles of creation significantly accelerates capability building.

\section{MORE DISTRIBUTED PARTICIPANTS}

As the global Internet infrastructure expands, talented individuals and specialized firms can contribute to creation efforts regardless of where they reside. For example, open source software initiatives embrace contributors from around the world. Global creation nets have certainly existed in the past, but now the interactions among the participants can be much more frequent and much richer, making it easier for distributed participants to contribute more actively to creation initiatives. For example, big wave surfers were previously forced to wait until the next surf competition or the next trip to a big wave location to share new practices. Now, with web cameras, video conferencing, and online discussion forums, big wave surfers can watch each other in action and discuss the relative merits of new approaches on a real-time basis. ${ }^{\mathrm{xi}}$

Participants in creation nets can be more widely distributed across institutions as well as geographies. The Internet and related technologies like web services with their open standards are making it easier for participants to use technology to enhance their communication across institutional boundaries. In the early days of information technology, establishing connections across institutional boundaries was a daunting task. The complexity involved both in establishing and managing these connections significantly limited the number of institutions that could be brought into these digital networks. Now, that has all changed, not just in terms of basic services like e-mail and document exchange, but also for much richer shared workspaces like wikis and other social media.

\section{MORE DISTRIBUTED RESOURCES}

Not only can the participants in creation nets operate more effectively from distributed locations, but the resources they need to support their creation tasks can become much more distributed as well. Building upon the early days of the Internet when researchers could access powerful computing resources remotely, sophisticated analytical and experimental tools like electron microscopes or powerful radio telescopes can now be shared and accessed from remote locations.

As previously mentioned, these technologies also enable sophisticated "pull" platforms to emerge, so that participants can readily search for and obtain the resources they need on demand rather than specifying their 
requirements in advance. These resources can be accessed quickly and cost-effectively, helping to accelerate creation activity.

\section{MORE RAPID ITERATIONS}

These technology platforms and tools are also helping to build mechanisms for rapid iteration. As mentioned in the surfing example above, richer and more versatile forms of distributed communication available on the Internet make it possible to comment more rapidly on the practices and products of other participants in creation networks. The availability of richer social software and video communication makes it possible for participants to better see the practices of others through visual renderings. These visual renderings help to create a sense of copresence and to support thicker forms of coordination. In the case of digital products, the participants can actually use these products as soon as they are created and provide much more rapid feedback to the creators of the products.

Participants in highly distributed creation nets more systematically explicate practices (although, of course, these practices can never be fully explicated) through such techniques as documentation, testing logs or linking structures to make it feasible for other participants to replicate practices in remote locations. The products of this explication can be more quickly accessed and tested by participants of the network, accelerating the replication of practice. Explicit reputation mechanisms help distributed participants to identify and connect more quickly with other participants with relevant expertise and provide rapid feedback regarding the performance of participants.

\section{ACCELERATING LEARNING AND CAPABILITY BUILDING}

On multiple levels, these technology platforms and tools are helping creation nets to become even more powerful engines of learning and capability building. First, by more effectively connecting distributed and highly motivated talent, these technologies amplify the potential for learning by exposing participants to a much greater diversity of experience and perspectives, one that's anchored in a context of specific issues and a pressing requirement for choice and action.

Second, these technologies allow greater scalability, connecting more participants together. As the networks incorporate more participants, they create opportunities for dynamic specialization. Participants can specialize in their areas of greatest strength, focusing on building capability in areas where they have the greatest potential rather than getting distracted by the need to perform a broader array of activities. Specialization thus becomes a precursor to more rapid learning and growth.

Third, a larger number of participants make it easier to support parallel problem-solving so that a broader range of options can be pursued and tested. Teams can get more rapid feedback on their efforts and benchmark their performance relative to other teams so that they can quickly learn from the experiences of others and adapt their efforts accordingly.

Fourth, the process of integrating the contributions of more distributed participants into consistent or compatible releases or offerings provides a significant opportunity for "productive friction" to generate additional insight and learning. With clear action points and demanding performance requirements, creation nets can use technology to bring distributed participants together more regularly and effectively around shared objects like models or drawings to engage on potential incompatibilities or inconsistencies in their creation efforts.

Fifth, the emphasis on the rapid appropriation and reuse of the knowledge, products, or services created establishes tight feedback loops and platforms for cumulative creation so that participants can rapidly build on the contributions of others and extend them in new directions.

Sixth, technology is making it easier to integrate into creation nets a growing segment of pro-amateurs who are passionate about particular areas of creation and willing to invest the time and effort required to develop deeper talent and discipline. Amateurs and professionals begin to develop complementary roles - amateurs refining certain dimensions of practice and professionals contributing theory and architecture to focus and give more meaning to the 
efforts of amateurs. In this way, the efforts of both professionals and amateurs are amplified and learning is accelerated on both sides

As these dynamics play out, creation nets are likely to proliferate and expand even more rapidly because of the increasing attractiveness to individuals and institutions of participating - and increasing penalties associated with not participating.

\section{CHALLENGES POSED BY CREATION NETS}

While very powerful, creation nets do not map readily to our existing institutional architectures. In many cases, executives are not even aware of the creation nets in which individuals within their organization are already participating, much less the creation nets that could augment their own internal capability-building efforts. Leadership teams need to identify existing creation nets and determine if there are opportunities to catalyze new ones to help accelerate relevant innovation and talent development. For each of these creation nets, leadership teams need to be clear what role(s) their institutions will play in the network - will they be an orchestrator, aggregator, or participant? They then need to reconfigure their institutions to enable them to play these roles more effectively

Today's management and leadership practices will often make it difficult to harness the full potential of creation nets. Our institutions have largely been organized around push models of resource mobilization. Effective participation in creation nets requires a different skill set focused on building, deploying, and managing pull models of resource mobilization.

Management within our institutions tends to focus on top-down models of direction within a framework of compensation and advancement policies specific to that institution. Participation within creation nets requires much greater reliance on distributed incentive structures where intrinsic incentives play a greater role than inside most institutions and where the extrinsic incentives tend to focus more heavily on non-cash incentives like the opportunity to accelerate capability building. In fact, creation nets tend to bring intrinsic and extrinsic motivations together in powerful ways. The desire to get better faster by working together with others works at both the individual level and the institutional level.

Institutions will need to reassess and redefine their intellectual property policies and practices in order to participate effectively in creation nets. As discussed earlier, institutions will need to become much more selective in their efforts to protect existing stocks of knowledge and much more adept in using their stocks of knowledge to contribute more actively in creation nets and to plug into promising flows of knowledge.

At a more fundamental level, leadership teams will need to challenge conventional mindsets within their own group and within their institutions more broadly. A natural reaction to accelerating change is to turn inward and tighten control in an attempt to protect what already has value. Creation nets require a different mindset, one that recognizes that flows of knowledge across institutional boundaries are the key to generating the new knowledge and new practices required to succeed in a rapidly changing world. This in turn requires an even more fundamental mindset shift beyond focusing on narrow efficiency gains and recognizing that the key to survival is to gain sufficient flexibility to embrace uncertainty and explore the possibilities that uncertainty creates. Leadership teams will need to be clear about what they want to achieve by participating in creation nets and continually reassess their goals in light of changing circumstances.

On a public policy dimension, the growth of creation nets will create significant challenges for nations and regions seeking to attract, retain, and enhance the productivity of their creative talent. Creative talent is becoming the source of comparative advantage, displacing more conventional structural advantages like climate, natural resources, or size of domestic markets. The global geography of creative capital is becoming much more fluid as the creative classes seek out the most hospitable locations and harness the power of the Internet and related technologies to connect with others in ways that accelerate their talent development. Creation nets will reach out and connect to this talent wherever it resides. 
The good news is that all locations become viable as a home for creative talent given the capabilities of creation nets to reach out and mobilize talent in distributed creation initiatives. The bad news is that urban areas around the world are competing to attract creative talent. New York and Silicon Valley must increasingly contend with new centers of creative talent like Shanghai and Bangalore. Talent will develop more rapidly if it becomes embedded in specialized local business ecosystems. These local business ecosystems in turn will thrive more if their participants connect with others around the world through creation nets.

To compete effectively for this talent, policy-makers will need to re-conceive public policy through the lens of accelerating talent development. This is not just a question of reassessing educational policies. Countries must reexamine all policies that affect the growth of local business ecosystems, including immigration and financial market regulation, as well as the ability of these ecosystems to connect into relevant creation nets, including such policies as intellectual property protection and trade regulation.

The proliferation and evolution of these creation nets sets the stage for movement to a positive sum game so that nations and regions can bootstrap off each other and accelerate their own talent development - but only if they understand the game and master the techniques required to play.

\section{MANAGEMENT TECHNIQUES TO BUILD SUCCESSFUL CREATION NETS}

Creation nets don't just emerge and evolve. They are shaped and governed by network organizers. Most analysts of open innovation spend very little time examining the governance mechanisms required to support sustained innovation, especially when a large number of participants are involved. Yet, successful creation nets require a deep understanding of the management techniques required to effectively deliver reliable performance to the marketplace.

Four management techniques are critical to the success of creation nets: choosing appropriate coordination approaches; balancing local innovation with effective integration; designing effective action points; and establishing effective performance feedback loops.

\section{Choosing Appropriate Coordination Approaches}

We made a distinction earlier between practice networks and process networks, depending on the degree of diversity of participants in the creation net. Understanding this distinction is important because the most effective coordination approaches will differ depending on which sub-set of creation nets is involved. Because of the diversity of participants, the organizers of process networks play a more active role in mobilizing participants. Specifically, they play an orchestration role - recruiting specific participants into the network and then deciding who will be involved in each creation initiative, the specific role they will play, and the performance requirements they must deliver.

In contrast, the coordination approach in practice networks is much looser, both in terms of recruitment and management of specific creation initiatives. Practice orchestrators tend to focus their coordination activity on the integration stage of the creation process when the contributions of the participants must be brought together and incorporated into a consistent or compatible release.

\section{Balancing Local Innovation With Effective Integration}

Creation nets of all types exhibit an important balance between looser and tighter forms of management. To understand how to strike the right balance, it is important to differentiate three primary challenges in the creation process:

- $\quad$ how to access and develop highly distributed talent

- how to provide appropriate contexts for these capabilities to come together and engage in collaborative experimentation, tinkering, and innovation

- how to effectively integrate the creations of diverse participants into shared "releases" 
In looking at how creation nets address each of these challenges, one can begin to discern interesting blends of emergent and managed behavior. Managed behavior is most pronounced at the integration level when the contributions of distributed participants must ultimately come together into a consistent or compatible release or offering. This is where governance structures become critical to resolve often deeply held differences. It is also where some of the greatest insight and innovation occurs as diverse parties clash and seek to address each other's concerns. In contrast, talent aggregation and development activities tend to be shaped by more emergent behavior, especially on the periphery of creation nets. Creation nets often rely on much more loosely organized environments like local business ecosystems or online forums to serve as attraction points for identifying and bringing together talent.

\section{Designing Effective Action Points}

Creation net organizers play their most active role at the integration stage. In fact, the success of creation nets hinges on using this integration stage as an action point to focus and align the efforts of diverse participants. At some point, participants must come together and either hand off their creations for others to build on or integrate their various creations into a consistent or compatible release - it is at this point that the rubber truly hits the road. By specifying when these actions must occur, the performance requirements that each participant must meet and the protocols for escalating and resolving disputes, network organizers create the institutional mechanisms necessary for productive friction to occur. We have discussed productive friction in much more detail in our book The Only Sustainable Edge.

The essential point is that diverse participants are forced to confront and resolve significant differences in approach. Rather than specifying the outcomes in terms of detailed blueprints, the designers of effective action points focus on specifying high level performance requirements and give the participants substantial degrees of freedom in determining how to meet these performance requirements. Greater freedom means greater opportunity for divergence, especially given the opportunity for parallel innovation initiatives by many participants made possible by modular management approaches. At the same time, by specifying timing and broader performance requirements, network organizers ensure that participants also come together and deliver functioning products to the marketplace.

\section{Establishing Effective Performance Feedback Loops}

While creation nets employ much looser management techniques relative to more traditional approaches to open innovation, these networks operate successfully in some of the most demanding global markets imaginable, from apparel to enterprise software. Loose management does not mean sloppy performance. On the contrary, these creation nets exhibit very high performance. More importantly, they are continually improving their performance at a faster pace than conventional enterprises can match.

How do they do this? In part, as discussed earlier, the answer hinges on the tight focus on relevant performance requirements . But there is something more. Successful creation nets build-in explicit performance feedback loops to give participants much greater visibility in how they are doing. Even in relatively loosely organized open source software initiatives, participants receive rapid feedback from others who have used their software. Broad adoption of software modules is one of the key drivers of status in these projects. Participants carefully monitor this measurement of performance, not only for their own software contributions but for the contributions of others and they strive to learn from programmers whose modules gain much greater acceptance.

\section{MOBILIZING EFFECTIVE CREATION NETS}

As should be clear by now, creation nets don't just emerge spontaneously, they are catalyzed and shaped by network organizers. As companies begin seeking to harness the benefits of creation nets, senior management needs to focus their own efforts to ensure the greatest impact. 


\section{Choose An Initial Arena To Build Creation Net Capability}

Not all areas of the business will benefit equally from creation nets. Focus initially on the parts of the business with three attributes: uncertain demand; the need for participation by many different specializations for creation activity to occur; and rapidly changing performance requirements in the marketplace. In these arenas creation nets have their most distinctive value - the ability to flexibly and scalably mobilize dispersed and diverse talent for innovation.

\section{Choose Whether To Establish A New Creation Net Or To Participate In An Existing Network}

Creation nets are still in a relatively early stage of development in many industries. Nonetheless, creation nets are already well established in a surprising array of industries ranging from apparel to motorcycles to software. Management needs to develop a clear view of the current level of creation net activity within relevant arenas. Where creation nets are already active, executives need to choose whether to participate in existing networks or become an organizer of a new creation net. Too often, the instinct will be to create a new network without fully recognizing the challenges involved. Creation nets have powerful network effects that make it more difficult for late entrants to come in and compete effectively for the attention and resources of relevant participants.

In part, the decision will depend on management's choices regarding the capabilities that will be required for them to maintain distinctiveness in the marketplace. For example, a high tech company may decide that their real distinctiveness will be a deep understanding of customer needs, and the relationships they can build with customers based on this understanding. In this case, the company may be well served by contracting with the organizer of an existing creation net to source new product designs. Even if the company decides that product design will be a continuing source of distinctiveness, there may be strong benefits to joining an existing creation net, especially if that creation net incorporates the company's products into broader systems or OEM equipment.

Before striking out and seeking to establish a new creation net, executives should be diligent in interviewing participants in existing creation nets to determine levels of satisfaction. They will need to use insight from these interviews to develop clear and compelling value propositions to attract participants to their competing creation net.

\section{Choose What Activities To Shed}

Effective participation in a creation net, whether as an organizer or participant, often hinges on the ability to focus and minimize potential competitive overlaps. For example, many Western high tech firms are sourcing new product designs from creation nets based in Asia while at the same time supporting their own product design initiatives. Since the organizers of these creation nets will be wary of competition from these high tech firms, they will be reluctant to bring these companies fully into product design innovation discussions. Equally if a high tech systems company chooses to establish a creation net, but is also involved in the design and manufacture of key subcomponents of those systems, it will likely have a difficult time convincing competing manufacturers of these subcomponents to participate in their creation net. Where these potential competitive overlaps exist, companies would be well-served to articulate as clearly as possible where they will choose to compete so that other participants can become more comfortable in deciding how much to share in collaborative innovation activities.

Shedding activities that can be more effectively performed by other participants in a creation net will also help a company focus its own resources on the activities where it can be truly distinctive. By avoiding distractions, it can accelerate its own capability building and get maximum benefit from its creation nets.

\section{Choose Appropriate Governance Models When Seeking To Become A Creation Net Organizer}

If a company chooses to become a creation net organizer, it must carefully think through the appropriate governance model to employ. As discussed earlier, this will depend upon where in the spectrum the participants in this creation net will fall, from highly similar to highly diverse practices. Is this creation net more of a practice 
network or a process network? The answer to this question will determine the nature and extent of coordination activity that the organizer will have to perform.

\section{Stage Initiatives In Establishing A Creation Net When Seeking To Become A Creation Net Organizer}

Creation nets evolve over time. Creation net organizers should resist the temptation to design large creation nets at the outset. Carefully pick a limited number of initial participants based on their depth of expertise and their openness to collaboration across enterprise boundaries. Existing trust-based relationships are the most desirable, but if these do not already exist, concentrate on deploying techniques to accelerate the building of trust and deepening of relationships. Define a minimal set of participation protocols at the outset and enlist the support of network participants in refining these protocols over time. As the organizer develops more experience with the looser management techniques characteristic of creation nets, expand the number of participants.

\section{Establish Appropriate Internal Mechanisms To Ensure Effective Participation In A Creation Net}

Whether a company chooses to become a creation net organizer or simply a participant, senior management must work hard to ensure that there is an effective two-way flow across their enterprise boundaries with other participants in the network. Line managers within the relevant business areas of the firm should be recruited to serve as champions and key liaisons with other participants in the creation net.

Two key risks exist. On the one hand, internal managers must resist the temptation to define their role within the creation net as pure "takers," scouring the network for relevant ideas, products, or capabilities while resisting efforts to share their own resources with other network participants. This may work in the very short-term but it will quickly lead to deterioration in value as other network participants begin to realize the one-way nature of the relationship. Internal managers must be very aggressive in asking "what value can we provide to the network?" in addition to "what value can the network provide us?" It is all too common to focus only on the latter question.

On the other hand, many enterprises are burdened with a not-invented-here-mindset that will make it difficult for managers to tap into the value that creation nets can provide. They will always be more comfortable with, and therefore prefer, internal resources. In these situations, senior executives must be active in communicating the value that creation nets can provide and establishing appropriate performance measurement and incentive mechanisms to reward management for effectively participating in creation nets.

\section{SUMMING IT UP}

We all know that open innovation can improve business performance, yet we continue to be frustrated by the large and persistent gap between potential and results. To the extent that open innovation occurs at all, it tends to be episodic and marginal to the overall performance of the enterprises. To be sustained and amplified, open innovation requires a specific set of institutional mechanisms. The institutional mechanisms underpinning creation nets represent the most powerful innovations of all - these innovations spawn wave upon wave of process and product innovations.

Creation nets offer an opportunity to amplify the performance impact from open innovation, but the opportunity can only be grasped if executives challenge existing mindsets. To harness the potential of creation nets executives will also need to master distinctive management techniques. Those who master these techniques will enjoy an unparalleled opportunity to rapidly improve performance and, at long last, close the gap that has made open innovation a seductive mirage for many and an exercise in frustration for others.

\section{Endnotes}

i In an email to the authors on September 28, 2004, Bill Joy indicated that he made this statement many times in speeches throughout the 1980s. 
ii Chesbrough, Henry William (2003), Open Innovation: The New Imperative for Creating And Profiting from Technology. (Boston: Harvard Business School Press)

iii Hagel III, John and Brown, John Seely, "From Push to Pull--Emerging Models for Mobilizing Resources," Journal of Service Science, Volume 1, Number 1, July 2008.

iv Hagel, John III and Brown, John Seely (2005), The Only Sustainable Edge. (Boston: Harvard Business School Press).

v Brown, John Seely and Duguid, Paul (2000)., The Social Life of Information, Boston: Harvard Business School Press.

vi Hagel, John III and Brown, John Seely (2005), The Only Sustainable Edge. (Boston: Harvard Business School Press).

vii Von Hippel, Eric (2005), Democratizing Innovation, (Cambridge: The MIT Press).

viii Prahalad, C.K., C.K. and Ramaswamy, Venkat (2004), The Future of Competition: Co-Creating Unique Value with Customers. (Boston: Harvard Business School Press).

ix Sawhney, Mohanbir and Prandelli, Emanuela, "Communities of Creation: Managing Distributed Innovation in Turbulent Markets," California Management Review, July 1, 2000.

x Weber, Steven (2004), The Success of Open Source. (Cambridge: Harvard University Press)

xi Hagel III, John and Brown, John Seely, "Innovating on the Edge of Big Waves," businessweek.com http://www.businessweek.com/innovate/content/jan2008/id20080130_724732.htm

\section{REFERENCES}

1. Brown, John Seely and Duguid, Paul (2000)., The Social Life of Information, Boston: Harvard Business School Press.

2. Hagel, John III and Brown, John Seely (2005), The Only Sustainable Edge. (Boston: Harvard Business School Press)

3. _Chesbrough , Henry William (2003), Open Innovation: The New Imperative for Creating And Profiting from Technology. Boston: Harvard Business School Press 